\title{
IMID-Biobank: Biobank of immune-mediated inflammatory diseases (IMIDs)
}

\author{
S Marsal $^{1 *}$, J Cañete ${ }^{2}$, J Tornero ${ }^{3}$, A Fernandez-Nebro ${ }^{4}$, J M Hernanz ${ }^{5}$, J Panes ${ }^{6}$, A Julià ${ }^{1}$, R Tortosa ${ }^{1}$ \\ From 5th European Workshop on Immune-Mediated Inflammatory Diseases \\ Sitges-Barcelona, Spain. 1-3 December 2010
}

\section{Objective}

Genomic analysis technologies are enabling an unprecedented characterization of human molecular variability. This high analytical power will be essential to meet the challenge of personalized medicine. More than ever, the quality of the biological samples will be essential to make the most of these new technologies. In order to obtain a large collection of samples from patients with Immune Mediated Inflammatory Diseases (IMIDs), the IMID-Biobank was created in 2008.

\section{Methods}

The IMID-Biobank is located in the Scientific Park of Barcelona. In collaboration with the Spanish National DNA Bank (www.bancoadn.org) different working protocols were implemented, regarding the collection, processing and storage of the IMID samples. The IMID-Biobank is itself an operating infrastructure that consists of a steering unit, a technical coordinator, a team of laboratory technicians and quality control unit. The purpose of the biobank, to provide samples of high quality, depends on the proper coordination of each of these elements. For this purpose, the IMID-Biobank has implemented a quality management system that allows continuous evaluation of all processes and procedures that take place in the biobank.

\section{Results}

The IMID-Biobank has collected, processed and stored samples from more than 8,000 patients with IMID diseases. Taking into account that from blood samples different products like genomic DNA, RNA, plasma or immortalized cell lines can be obtained, the IMID-Biobank is actually storing more than 50,000 products derived from
IMID patients. The IMID-Biobank is the first Spanish biobank associated with a hospital that obtains the ISO 9001:2000 quality certificate.

\section{Conclusions}

The existence of biobanks like IMID-Biobank will be essential to make the most of genomic technologies and finally meet the challenge of personalized medicine.

\section{Author details \\ ${ }^{1}$ Institut de Recerca Hospital Vall Hebron, Grup Recerca de Reumatologia, Barcelona, Spain. ${ }^{2}$ Hospital Clinic i Provincial, Servei Reumatologia, Barcelona, Spain. ${ }^{3}$ Hospital Universitario de Guadalajara, Servicio Reumatologia, Guadalajara, Spain. ${ }^{4}$ Hospital Carlos Haya, Servicio Reumatologia, Malaga, Spain. ${ }^{5}$ Hospital La Princesa, Servicio Dermatologia, Madrid, Spain. ${ }^{6}$ Hospital Clinic i Provincial, Servei Gastroenterologia, Barcelona, Spain.}

Published: 25 November 2010

doi:10.1186/1479-5876-8-S1-P6

Cite this article as: Marsal et al:: IMID-Biobank: Biobank of immunemediated inflammatory diseases (IMIDs). Journal of Translational Medicine 2010 8(Suppl 1):P6. 